\title{
Submicroscopic Plasmodium prevalence in relation to malaria incidence in 20 villages in western Cambodia
}

Rupam Tripura ${ }^{1,2^{*}+}$ (D, Thomas J. Peto ${ }^{1,3+}$, Christianne C. Veugen ${ }^{2}$, Chea Nguon $^{4}$, Chan Davoeung ${ }^{5}$, Nicola James ${ }^{6}$, Mehul Dhorda 1,7, Richard J. Maude 1,3,8, Jureeporn Duanguppama ${ }^{1,9}$, Krittaya Patumrat ${ }^{1,9}$, Mallika Imwong ${ }^{1,9}$, Lorenz von Seidlein 1,3, Martin P. Grobusch ${ }^{2}$, Nicholas J. White ${ }^{1,3}$ and Arjen M. Dondorp ${ }^{1,3}$

\begin{abstract}
Background: Cambodia has seen a marked reduction in the incidence of Plasmodium falciparum over the past decade without a corresponding decline in Plasmodium vivax incidence. It is unknown to what extent local transmission is sustained by a chain of clinical and sub-clinical infections or by continued re-introduction via migration. Using an ultrasensitive molecular technique, 20 villages in western Cambodia were surveyed to detect the low season prevalence of $P$. falciparum and $P$. vivax and local treatment records were reviewed.
\end{abstract}

Methods: During March to May 2015 cross-sectional surveys were conducted in 20 villages in Battambang, western Cambodia. Demographic and epidemiological data and venous blood samples were collected from 50 randomly selected adult volunteers in each village. Blood was tested for Plasmodium infections by rapid diagnostic test (RDT), microscopy and high volume ( $0.5 \mathrm{ml}$ packed red blood cell) quantitative polymerase chain reaction (UPCR). Positive samples were analysed by nested PCR to determine the Plasmodium species. Malaria case records were collected from the Provincial Health Department and village malaria workers to determine incidence and migration status.

Results: Among the 1000 participants, 91 (9.1\%) were positive for any Plasmodium infection by uPCR, seven (0.7\%) by microscopy, and two (0.2\%) by RDT. uPCR P. vivax prevalence was 6.6\%, P. falciparum $0.7 \%$, and undetermined Plasmodium species 1.8\%. Being male (adjusted OR 2.0; $95 \% \mathrm{Cl} 1.2-3.4$ ); being a young adult $<30$ years (aOR 2.1; $95 \% \mathrm{Cl} 1.3-$ 3.4); recent forest travel (aOR 2.8; 95\% Cl 1.6-4.8); and, a history of malaria (aOR 5.2; 95\% Cl 2.5-10.7) were independent risk factors for parasitaemia. Of the clinical malaria cases diagnosed by village malaria workers, $43.9 \%$ (297/634) and 38.4\% (201/523) were among migrants in 2013 and in 2014, respectively. Plasmodium vivax prevalence determined by UPCR significantly correlated with vivax malaria incidences in both 2014 and 2015 ( $p=0.001$ and 0.002, respectively), whereas no relationship was observed in falciparum malaria ( $p=0.36$ and $p=0.59$, respectively).

Discussion: There was heterogeneity in the malaria parasite reservoir between villages, and Plasmodium prevalence correlated with subsequent malaria incidence. The association was attributable chiefly to $P$. vivax infections, which were nine-fold more prevalent than $P$. falciparum infections. In the absence of a radical cure with 8-aminoquinolines, $P$. vivax transmission will continue even as P. falciparum prevalence declines. Migration was associated with over a third of incident cases of clinical malaria.

Trial registration clinicaltrials.gov (NCT01872702). Registered 4 June 2013

\footnotetext{
*Correspondence: Rupam@tropmedres.ac

${ }^{\dagger}$ Rupam Tripura and Thomas J. Peto contributed equally to this work

${ }^{1}$ Mahidol Oxford Tropical Medicine Research Unit, Faculty of Tropical

Medicine, Mahidol University, 420/6 Rajvithi Rd, Rajthevee,

Bangkok 10400, Thailand

Full list of author information is available at the end of the article
} 
Keywords: Malaria, Plasmodium Falciparum, Plasmodium vivax, Asymptomatic, Sub-clinical, Sub-microscopic, uPCR, Cambodia

\section{Background}

Malaria incidence in Cambodia has decreased by approximately 42\% from 2009 to 2015 [1]. The treatment records of the National Malaria Control Programme, which do not include the private sector, report that this was driven chiefly by a decline of $55 \%$ in Plasmodium falciparum, whereas Plasmodium vivax declined by only $17 \%$. This progress was due to the roll-out of rapid diagnostic tests (RDTs), treatment of confirmed malaria cases with artemisinin combination therapy (ACT) provided by village malaria workers (VMWs), and the distribution of insecticide treated bed-nets (ITNs) [1].

The Thai-Cambodian border area around Pailin Province in western Cambodia has been a cradle of anti-malarial drug resistance. In the 1960s, resistance of $P$. falciparum to chloroquine emerged from this area before spreading to Asia and Africa and causing millions of deaths [2]. Since 2007 several studies have shown that $P$. falciparum in western Cambodia has become resistant to artemisinins and more recently to the partner drugs used in ACT, such as piperaquine [3-6]. Subsequently, artemisinin-resistant Plasmodium parasites were found on the Thai-Myanmar border and other parts of Southeast Asia [7]. ACT offers the most effective anti-malarial drugs currently available, and the loss of these drugs would be a setback of potentially disastrous proportions for global malaria control and for elimination strategies in Southeast Asia [8, 9]. There is increasing evidence that in order to prevent the spread of artemisinin-resistant $P$. falciparum it is necessary to completely interrupt $P$. falciparum transmission [10-12].

Previous surveys have described an asymptomatic reservoir of malaria in Cambodia [13, 14]. Microscopy and RDTs are not sensitive enough to detect the majority of sub-clinical Plasmodium infections [13, 15, 16]. Highvolume, ultra-sensitive, quantitative polymerase chain reaction ( $\mathrm{uPCR}$ ) is a recently developed method that can detect approximately $85 \%$ of all sub-clinical Plasmodium parasitaemias and thus characterize in detail the microepidemiology of malaria in areas of low transmission. These sub-clinical parasitaemias may play an important role in the transmission of infection, especially in areas of low and seasonal transmission $[17,18]$.

There are large seasonal population movements in and out of Samlout District in Battambang Province where the study was conducted. People from the district, predominantly men, spend time working outside the district and some return infected with malaria parasites. Agricultural labourers from other provinces come into the district to work during harvests and may harbour sub-clinical malaria infections acquired in other parts of the country. Seasonal work in forests collecting fruit, vines and hunting small game puts local people at risk of infection. These may contribute disproportionately to the transmission of malaria parasites. Battambang Provincial Health Department reports that approximately 83\% of all malaria cases were aged $15-49$ years, and $82 \%$ were males in 2014. The proportion of malaria cases among migrant workers in Battambang increased from 19\% in 2009 to around 40\% in 2014. In Cambodian border areas, the role of local, national and international migrants in malaria transmission has been acknowledged; however, the attributable fraction of infections has not been determined [19]. Outdoor and forest-acquired malaria infections continue to be important sources of transmission despite high rates of deforestation in the border areas of Cambodia.

Accurate malaria risk stratification is important for targeting control and elimination activities. Following the decline in $P$. falciparum infections over the past decade in western Cambodia, it is unknown to what extent local transmission is sustained by a chain of clinical infections, a reservoir of sub-microscopic malaria, or by continued introduction via migration. 20 villages in western Cambodia were surveyed to detect the sub-clinical prevalence of Plasmodium infections in an area where transmission is low but persistent and analysed the data with reference to available treatment records.

\section{Methods}

Study site

During the low season of malaria in March to May 2015, cross-sectional surveys were conducted in 20 villages in Samlout District, Battambang Province, western Cambodia. All available VMW records and provincial malaria data were reviewed. Samlout District lies to the south of Pailin Province, north of Pursat Province and the Cardamom Mountains, and east of Thailand (Fig. 1). Samlout is primarily an agricultural district with forested areas along the border. Samlout was chosen for this study as it has villages with some of the highest incidence of clinical malaria in Battambang Province and seasonal transmission.

\section{Selection of villages and participants}

Within Samlout District, 18 villages with the highest malaria incidence in 2014 were selected along with 


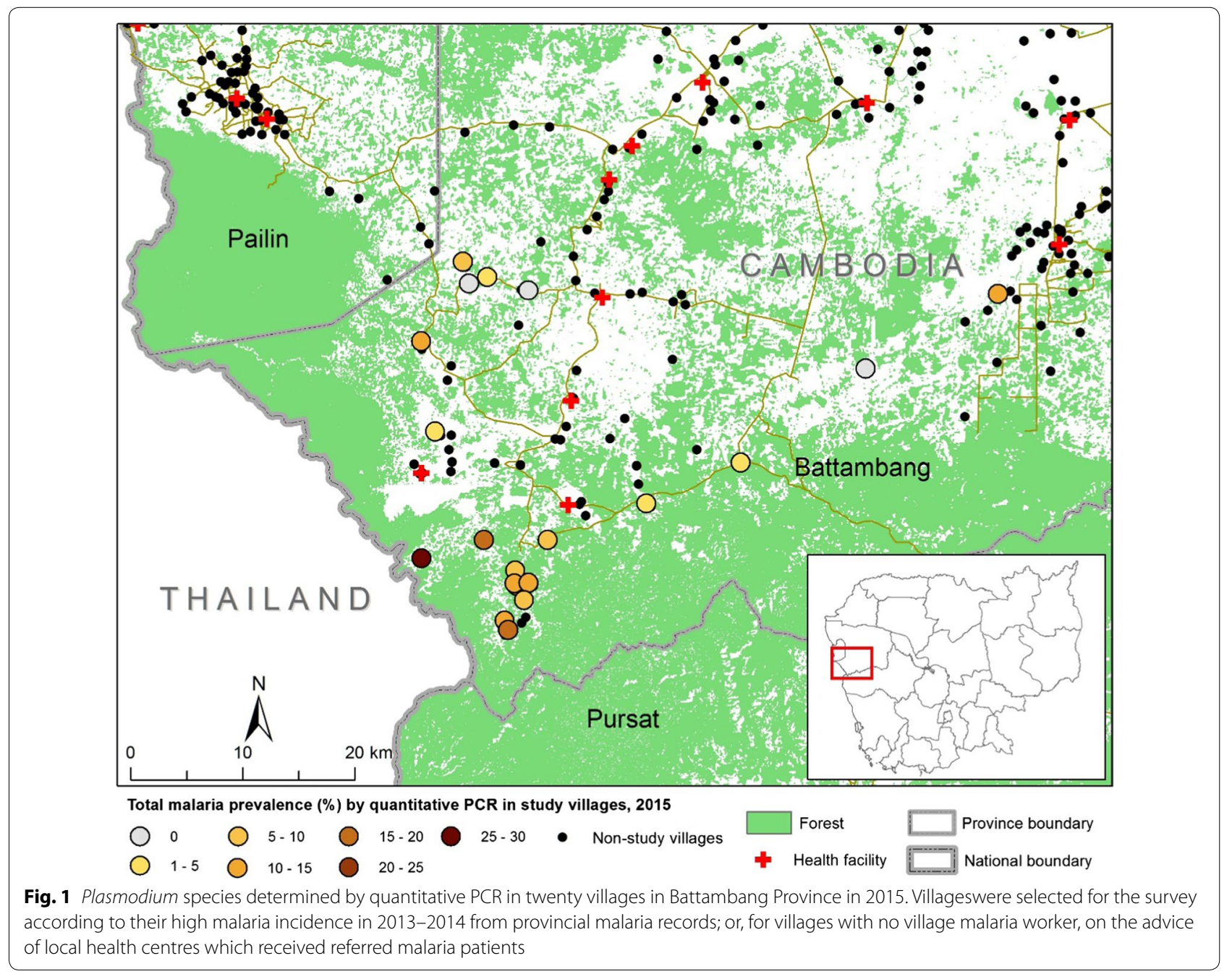

two neighbouring villages, totalling 20. A census based on pre-existing village population lists was updated and household lists were prepared. Surveyed households were selected using randomization methods from the list to ensure that every household had an equal chance of being selected. A stratified sampling method obtained approximately equal samples of adults aged 18 years or over from four groups (male/female, under/ over 30 years). Unless the total number of households in a village was fewer than 50 , no more than one person per household was selected. For the villages with fewer than 50 households, more than one participant was randomly selected from the list. In cases of refusal or a person being absent on the survey day, replacement participants were selected randomly from the same household or the next household on the list. Pregnant women were excluded from the randomization as they would be ineligible to join a mass anti-malarial treatment study which followed this survey.

\section{Community engagement and informed consent}

Public meetings were conducted in Khmer by a dedicated Community Engagement Team (CE-Team) to inform the commune authorities, VMWs and village leaders about the study. All residents were invited to a meeting prior to the survey where the consent form was read aloud and questions were answered. Individual written informed consent was obtained immediately prior to sample collection and interview. A fingerprint was obtained from participants who could not read and write and a witness countersigned their consent form.

\section{Survey methods}

Anthropometric and demographic data were collected, including tympanic temperature, height, weight, health status, malaria history, bed net use, travel history, and occupation; $1.5 \mathrm{ml}$ of venous blood was collected in an EDTA tube. Samples were kept in a cool box and sent to a nearby hospital laboratory on the same day. If a 
participant was febrile (defined as tympanic temperature $\geq 37.5 \mathrm{C}^{\circ}$ ) an RDT (SD BIOLINE Malaria Ag P.f/P.v, Standard Diagnostics, Inc, Gyeonggi-do, Republic of Korea) was performed immediately, and if positive, treatment was provided by the VMW according to national guidelines.

\section{Laboratory methods}

The samples were separated; $200 \mu$ of whole blood was used to prepare a dried blood spot on filter paper, a blood smear for microscopy, and to perform haemoglobin assay (HemoCue ${ }^{\circledR} \mathrm{Hb} 201^{+}$Analyzer SE-262 71 Angeltholm, Sweden). Blood was tested for malaria by RDT and microscopy (asexual parasites/500 white blood cells (WBCs)). The remaining blood was centrifuged and plasma, buffy coat and $500 \mu \mathrm{l}$ of packed red blood cells (pRBCs) for uPCR were separated into three different cryo-tubes and stored at $-80{ }^{\circ} \mathrm{C}$. Ten per cent negative controls of total participant samples were added for each village. All the samples were transported to the Mahidol University Faculty of Tropical Medicine molecular laboratory in Bangkok.

\section{Molecular laboratory procedures}

A detailed description, evaluation and validation of the high-volume ultrasensitive (uPCR) methodology has been reported previously [15]. In brief, batches of $500 \mu \mathrm{l}$ of pRBCs were thawed and the DNA template, for detection and quantification of Plasmodium by PCR, was extracted and purified. An ultrasensitive quantitative real-time PCR method was used to detect the presence of malaria parasites and to estimate the numbers (genomes) of parasite per millilitre from each sample. The $18 \mathrm{~S}$ rRNA-targeting primers and hydrolysis probes used in the assay have been validated and are highly specific for Plasmodium species. The lower limit of accurate quantitation of this method is 22 parasites $/ \mathrm{ml}$ of whole blood [15].

A Quanti-Tect Multiplex PCR No ROX ${ }^{\circledR}$ (QIAGEN, Hilden, Germany) was used, and the PCR mixture and the cycling conditions were as per manufacturer's instructions. For samples containing parasite DNA by uPCR analysis, Plasmodium species detection was attempted using real time PCR protocols specific to 18sRNA of $P$. falciparum and $P$. vivax. Samples for which Plasmodium species were not determined were reported as 'unknown species'.

To detect polymorphisms associated with reduced susceptibility to artemisinin derivatives the open-reading frame of the PF3D7_1343700 kelch propeller domain was amplified using a nested PCR protocol [7]. Purified PCR products were sequenced at Macrogen, Republic of Korea and analysed using BioEdit version 7.1.3.0., using the 3D7 kelch13 sequence as reference (Accession: XM_001350122.1).

\section{Statistical analysis and data management}

Data were collected on case record forms and entered on smartphones before being exported into Open Clinica. Survey data were analysed by univariate and multivariate methods to identify risk factors for parasitaemia. Odds ratios with $95 \%$ confidence intervals were calculated to explore which variables were independently associated with subclinical infection. The Pearson Chi squared test was used to test for the significance of associations. Variables that were independently associated with sub-clinical parasitaemia at the level of $\mathrm{p}<0.05$ were explored by logistic regression to produce adjusted odds ratios. Incidence rate was calculated by dividing total malaria cases in a given year with the total village population and multiplied by 1,000. Malaria incidence and prevalence were compared by linear regression. The analysis was done in STATA 14.0 (Timberlake, USA).

\section{Additional data sources and clinical malaria incidence}

Malaria case reports were obtained from the Provincial Health Department and VMW records. Available treatment records were reviewed by cross-checking with the VMWs to confirm the identity of patients. The incidence of clinical malaria for 2014 and 2015 was compared to the prevalence of malaria parasites detected by uPCR in March and April 2015 for each village. Villages with no Health Department records were excluded from this comparison. For villages where only a hamlet (Chakriya: CKA, Samlaut: SLT, Kompoung Tuk A: KTA and Kompoung Tuk B: KTB) was selected for the prevalence survey, the incidence rate of the whole village population was used for the analyses. All prevalence survey participants were from registered households and were considered to be residents.

\section{Results}

Description of the study population

Population size and malaria incidence in 2014-2015 differed significantly among the 20 study villages. Population size ranged from 147 (in Ou Nounong: ONG) to 1012 (in Ouda: ODA). Malaria incidence per thousand ranged between two and 224 among villages in 2014 and 2-166 in 2015. Two villages (CKA, Miko: MKO) in 2014 and two villages (CKA, Peam: PEM) in 2015 were not listed in Provincial Health Department records, so no incidence data were available for these villages (Table 1). The median age of the study participants was 33 years (range from 18 to 78 years). The median age of male and female participants were 34 years (18-72 years) and 32.5 years (17-78 years), respectively; $11.7 \%(117 / 1000)$ subjects had a temperature 
Table 1 Plasmodium species prevalence by uPCR in 2015 cross-sectional surveys and malaria incidence from 2014-15

\begin{tabular}{|c|c|c|c|c|c|c|c|c|}
\hline \multirow[t]{2}{*}{ Village code } & \multicolumn{4}{|c|}{ uPCR prevalence } & \multicolumn{4}{|c|}{ Clinical cases from health records } \\
\hline & $\begin{array}{l}\text { P. falciparum } \\
\text { n/50 } \\
(\%)\end{array}$ & $\begin{array}{l}\text { P. vivax } \\
\text { n/50 (\%) }\end{array}$ & $\begin{array}{l}{ }^{a} P \text { P. species } \\
\mathrm{n} / 50(\%)\end{array}$ & $\begin{array}{l}\text { Population } \\
2014-2015\end{array}$ & $\begin{array}{l}2014 \text { PF or mix } \\
\text { cases } \\
\text { (incidence } \\
\text { per 1000) }\end{array}$ & $\begin{array}{l}2014 \text { All } \\
\text { malaria cases } \\
\text { (incidence } \\
\text { per 1000) }\end{array}$ & $\begin{array}{l}2015 \text { Pf or mix } \\
\text { cases } \\
\text { (incidence } \\
\text { per 1000) }\end{array}$ & $\begin{array}{l}2015 \text { All malaria } \\
\text { cases } \\
\text { (incidence } \\
\text { per 1000) }\end{array}$ \\
\hline CKA & 0 & $13(26)$ & $2(4)$ & 409 & No record & No record & No record & No record \\
\hline ONG & 0 & $4(8)$ & $5(10)$ & 147 & $7(47.6)$ & $33(224.5)$ & $2(13.6)$ & $12(81.6)$ \\
\hline PTA & $1(2)$ & $5(10)$ & $2(4)$ & 283 & $7(24.7)$ & $39(137.8)$ & $0(0.0)$ & $2(7.1)$ \\
\hline PRY & 0 & $8(16)$ & 0 & 614 & $11(17.9)$ & $115(187.3)$ & $44(71.7)$ & $102(166.1)$ \\
\hline OTG & 0 & $5(10)$ & $2(4)$ & 239 & $10(41.8)$ & $43(179.9)$ & $11(46.0)$ & $26(108.8)$ \\
\hline TTK & 0 & $7(14)$ & 0 & 214 & $1(4.7)$ & $21(98.1)$ & $3(14.0)$ & $7(32.7)$ \\
\hline SLT & 0 & $5(10)$ & $1(2)$ & 657 & $11(16.7)$ & $43(65.4)$ & $38(57.8)$ & $61(92.8)$ \\
\hline CMN & 0 & $6(12)$ & 0 & 642 & $8(12.5)$ & $17(26.5)$ & $7(10.9)$ & $13(20.2)$ \\
\hline PEM & 0 & $4(8)$ & $2(4)$ & 257 & 0.0 & $1(3.9)$ & No record & No record \\
\hline OTT & $2(4)$ & $1(2)$ & $2(4)$ & 300 & 0.0 & $1(3.3)$ & $2(6.7)$ & $2(6.7)$ \\
\hline ODA & $3(6)$ & $1(2)$ & 0 & 1012 & $28(27.7)$ & 35 (34.6) & 19 (18.8) & $22(21.7)$ \\
\hline $\mathrm{OKH}$ & 0 & $2(4)$ & $1(2)$ & 631 & $3(4.8)$ & 45 (71.3) & $15(23.8)$ & $29(46.0)$ \\
\hline VRM & $1(2)$ & $2(4)$ & 0 & 291 & 28 (96.2) & $71(244.0)$ & $8(27.5)$ & $28(96.2)$ \\
\hline KTB & 0 & $1(2)$ & 0 & 748 & $5(6.7)$ & $10(13.4)$ & $1(1.3)$ & $3(4.0)$ \\
\hline $\mathrm{SRH}$ & 0 & $1(2)$ & 0 & 562 & $3(5.3)$ & $6(10.7)$ & $2(3.6)$ & $3(5.3)$ \\
\hline MKO & 0 & 0 & $1(2)$ & 313 & No record & No record & $3(9.6)$ & 15 (47.9) \\
\hline $\mathrm{OCL}$ & 0 & $1(2)$ & 0 & 456 & $0(0.0)$ & $1(2.2)$ & $3(6.6)$ & $5(11.0)$ \\
\hline CLK & 0 & 0 & 0 & 275 & $3(10.9)$ & $6(21.8)$ & $2(7.3)$ & $3(10.9)$ \\
\hline KTA & 0 & 0 & 0 & 748 & $5(6.7)$ & $10(13.4)$ & $1(1.3)$ & $3(4.0)$ \\
\hline APP & 0 & 0 & 0 & 397 & $0(0.0)$ & $4(10.1)$ & $0(0.0)$ & $1(2.5)$ \\
\hline
\end{tabular}

Incidence total malaria cases/total village population* 1000

Prevalence total Plasmodium species detected by uPCR/total number of test* 100

a Positive for Plasmodium by high-volume qPCR, but the parasite genome count was too low for subsequent nested PCR to determine Plasmodium species

of $37.5{ }^{\circ} \mathrm{C}$ or higher; $65.4 \%(654 / 1000)$ had a history of malaria at any time prior to the survey.

\section{Prevalence of Plasmodium species by detection method}

Among 1000 participants, 91 (9.1\%) were positive for any Plasmodium species by uPCR, seven $(0.7 \%)$ by microscopy, and two $(0.2 \%)$ by RDT. By uPCR, P. vivax prevalence was 6.6\% (66/1000), P. falciparum 0.7\% (7/1000), and $1.8 \%(18 / 1000)$ had undetermined Plasmodium species. In Table 1 the parasite prevalence by uPCR is shown for each village.

The prevalence in the villages ranged from 0 to $30 \%$ by uPCR (test for heterogeneity $\mathrm{p}<0.001$ ). Plasmodium falciparum parasites were detected by UPCR in four of the 20 villages, with $P$. falciparum prevalence ranging from 2 to $6 \%(\mathrm{p}=0.010)$. Plasmodium vivax infections were detected by uPCR in 16 villages, with $P$. vivax prevalence ranging between 2 and 26\%, ( $<<0.0001)$ and the undetermined Plasmodium species were detected in nine villages, prevalence ranging from 2 to $10 \%$ ( $\mathrm{p}=0.011$ for heterogeneity). Three out of 20 villages with had no parasite prevalence by uPCR.

\section{Factors associated with Plasmodium species positivity} by UPCR

Factors associated with any positive Plasmodium species by uPCR are shown in Table 2. Plasmodium species prevalence among males was $14.1 \%(66 / 402)$ versus $4.7 \%$ (25/532) among females $\mathrm{p}<0.001 ; 11.5 \%$ (51/443) among those aged $18-29$ versus $7.2 \%$ (40/557) among those aged 30 or above $\mathrm{p}=0.018$. Plasmodium prevalence was $6.8 \%$ (8/117) among those with temperature of $37.5{ }^{\circ} \mathrm{C}$ or higher versus $9.3 \%(83 / 883)$ among those with a temperature below $37.5^{\circ} \mathrm{C}(\mathrm{p}=0.365)$. Among participants with previous history of clinical malaria, 12.5\% (82/654) were parasitaemic versus $2.6 \%(9 / 346)$ among those who said they had never had malaria ( $\mathrm{p}<0.0001)$.

Prevalence of Plasmodium parasites among participants spending one or more nights in a forest within the same period was $22.4 \%(35 / 156)$ versus $6.6 \%(56 / 844)$ among those without any forest travel $(\mathrm{p}<0.001)$. Among participants with parasitaemia, 27/91 (29.7\%) had recently stayed overnight in the forest in the same province, and $8 / 91(8.8 \%)$ had stayed in a forest in a different province; $56 / 91$ (62\%) did not have a history of overnight 
Table 2 Univariate associations between factors investigated for prevalence of Plasmodium infection by uPCR

\begin{tabular}{|c|c|c|c|c|c|c|}
\hline Variable & Number & $\begin{array}{l}\text { uPCR positive } \\
\text { for Plasmodium } \\
\text { Number (\%) }\end{array}$ & $\begin{array}{l}\text { uPCR negative } \\
\text { for Plasmodium } \\
\text { Number (\%) }\end{array}$ & $p$ value* & $\begin{array}{l}\text { Odds ratio }^{a} \\
(95 \% \mathrm{Cl})\end{array}$ & $\begin{array}{l}\text { Adjusted } \\
\text { odds ratio }^{b} \\
(95 \% \mathrm{Cl})\end{array}$ \\
\hline Number & 1000 & $91(9)$ & $909(91)$ & & & \\
\hline Gender & & & & $<0.0001$ & $\begin{array}{l}3.3 \\
(2.0-5.4)\end{array}$ & $\begin{array}{l}2.0 \\
(1.2-3.4)\end{array}$ \\
\hline Male & 468 & $66(14)$ & $402(86)$ & & & \\
\hline Female & 532 & $25(5)$ & $507(95)$ & & & \\
\hline Age groups (years) & & & & 0.018 & $\begin{array}{l}1.6 \\
(1.1-2.6)\end{array}$ & $\begin{array}{l}2.1 \\
(1.3-3.4)\end{array}$ \\
\hline $18-30$ & 443 & $51(11.5)$ & $392(88.5)$ & & & \\
\hline$>30$ & 557 & $40(7.2)$ & $517(92.8)$ & & & \\
\hline Tympanic temperature $\left({ }^{\circ} \mathrm{C}\right)$ & & & & 0.365 & $\begin{array}{l}0.7 \\
(0.3-1.5)\end{array}$ & $\begin{array}{l}0.9 \\
(0.4-1.9)\end{array}$ \\
\hline$\leq 37.5$ & 883 & $83(9)$ & $800(91)$ & & & \\
\hline$\geq 37.5$ & 117 & $8(7)$ & $109(93)$ & & & \\
\hline Self-reported fever in previous $48 \mathrm{~h}$ & & & & 0.255 & $\begin{array}{l}0.6 \\
(0.2-1.5)\end{array}$ & $\begin{array}{l}0.7 \\
(0.3-1.8)\end{array}$ \\
\hline Fever & 87 & $5(6)$ & $82(94)$ & & & \\
\hline No fever & 913 & $86(9)$ & $827(91)$ & & & \\
\hline $\begin{array}{l}\text { Self-reported illness in the previous } \\
48 \mathrm{~h}\end{array}$ & & & & 0.121 & $\begin{array}{l}0.7 \\
(0.5-1.1)\end{array}$ & $\begin{array}{l}0.9 \\
(0.6-1.5)\end{array}$ \\
\hline Illness & 506 & $39(8)$ & $467(92)$ & & & \\
\hline No illness & 494 & $52(11)$ & $442(89)$ & & & \\
\hline $\begin{array}{l}\text { Self-reported history of ever having } \\
\text { had malaria in past }\end{array}$ & & & & $<0.0001$ & $\begin{array}{l}5.3 \\
(2.6-10.9)\end{array}$ & $\begin{array}{l}5.1 \\
(2.5-10.7)\end{array}$ \\
\hline Yes & 654 & $82(13)$ & $572(87)$ & & & \\
\hline No & 346 & $9(3)$ & $337(97)$ & & & \\
\hline Forest visit in the previous 3 months & & & & $<0.0001$ & $\begin{array}{l}4.1 \\
(2.5-6.5)\end{array}$ & $\begin{array}{l}2.7 \\
(1.6-4.8)\end{array}$ \\
\hline Yes & 156 & $35(22)$ & $121(78)$ & & & \\
\hline No & 844 & $56(7)$ & $788(93)$ & & & \\
\hline Travel in the previous 3 months & & & & 0.489 & $\begin{array}{l}1.2 \\
(0.7-1.9)\end{array}$ & $\begin{array}{l}1.1 \\
(0.7-1.8)\end{array}$ \\
\hline Yes & 245 & $25(10)$ & $220(90)$ & & & \\
\hline No & 755 & $66(9)$ & $689(91)$ & & & \\
\hline Bed net use & & & & 0.004 & $\begin{array}{l}0.5 \\
(0.3-0.8)\end{array}$ & $\begin{array}{l}0.8 \\
(0.5-1.5)\end{array}$ \\
\hline Everyday & 849 & $68(8)$ & $781(92)$ & & & \\
\hline Sometimes or never & 151 & $23(15)$ & $128(85)$ & & & \\
\hline
\end{tabular}

* Pearson Chi Square test

a Mantel Haenszel odds

b Logistic regression, adjustment for gender, age group, temperature $\leq 37.5^{\circ} \mathrm{C}$

stay in the forest $(\mathrm{p}<0.001)$. Parasite prevalence among participants with a history of travel outside the village within the previous 3 months was $10.2 \%(25 / 245)$ versus $8.7 \%(66 / 755)$ among those without any travel history $(\mathrm{p}=0.489)$. Among those with parasites, $66.7 \%(66 / 91)$ did not travel in comparison to $27.3 \%$ (25/91) who travelled outside the village. Among the 25 positives who travelled outside the village, $44 \%(11 / 25)$ had stayed overnight in the forest.
Prevalence of parasitaemia was 8.0\% (68/849) among those who used ITNs every night, 15.2\% (20/132) among those who sometimes used ITNs, and $15.8 \%(3 / 19)$ among those who never used ITNs, $\mathrm{p}=0.004$. Parasite prevalence varied between the strata used for sampling: 19.9\% (39/196) among young men, 9.9\% (27/272) among older men, $4.8 \%(12 / 247)$ among young women, and $4.6 \%(13 / 285)$ among older women. Men were more likely to use ITNs irregularly or never $(27.1 \% ; 127 / 468)$ 
versus women $(24.5 \% ; 4 / 532) \mathrm{p}<0.0001$. Men were also more likely than women to stay overnight in forests $28.4 \%$ $(133 / 468)$ versus $4.3 \%(23 / 532), \mathrm{p}<0.0001$. Young men (35.2\%; 69/196) used ITNs irregularly or never, and 36.7\% (72/196) had spent a night in a forest within the past 3 months.

After adjustment, being male (OR 2.0; 95\% CI $1.2-3.5$ ), younger adults (age 18-30; OR 2.1; 95\% CI 1.3-3.4), recent forest travel (OR 2.6; 95\% CI 1.5-4.5), and a prior history of malaria (OR 5.3; 95\% CI 2.611.0) remained significantly associated with parasite prevalence.

Parasitaemia was higher among P. falciparum-positive individuals than those with $P$. vivax. Parasitaemia was lowest among people with undetermined Plasmodium species where the species were not determined. Tympanic temperature was not associated with the level of parasitaemia for any Plasmodium species (Fig. 2).

\section{Sub-clinical Plasmodium falciparum}

Among participants with Plasmodium parasitaemia, seven/91 had $P$. falciparum parasites. Among the seven with $P$. falciparum parasites, ages ranged from 22 to 49 years. None reported fever within the $48 \mathrm{~h}$ before screening and none had a temperature $>37.5{ }^{\circ} \mathrm{C}$. All reported having clinical malaria in the past, and five reported illness in the previous $48 \mathrm{~h}$. Three came from one village, ODA, and two from another, Ou Ta Teak (OTK). Four had stayed in a local forest overnight in the previous 3 months, of whom two reported travel outside the village in the same period. Two were positive both by RDT and by microscopy. Only one of seven falciparum positives had a history of travel to another province within the previous 3 months. Five reported everyday ITN use.

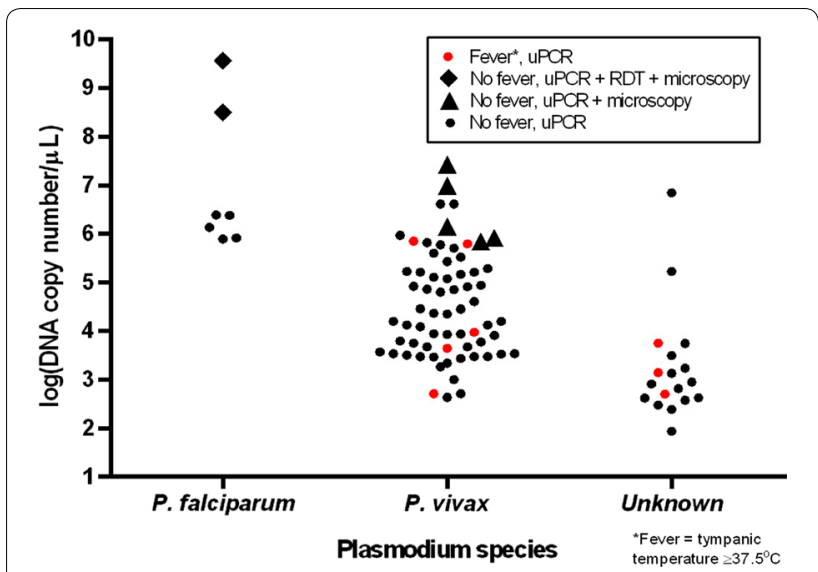

Fig. 2 Parasite genome count by Plasmodium species, tympanic temperature $\geq 37.5^{\circ} \mathrm{C}$ and method of detection

\section{Markers of artemisinin resistance}

Six of seven $P$. falciparum parasite strains detected by uPCR could be sequenced for PfKelch 13 mutations. All six P. falciparum isolates obtained Pfkelch13 C580Y mutation.

\section{Association between sub-clinical prevalence and clinical malaria incidence}

Malaria incidence was obtained for 19/20 survey locations from provincial health department records in 2014 and 2015. Overall malaria prevalence in the MarchApril 2015 survey measured by uPCR significantly correlated with the incidence in each of 2014 and 2015 ( $\mathrm{p}=0.003$ and $\mathrm{p}<0.001$ ), respectively. Plasmodium vivax prevalence correlated with $P$. vivax incidence $(\mathrm{p}=0.001$ and $\mathrm{p}=0.002)$, respectively. No correlation was observed between $P$. falciparum prevalence and incidence $(\mathrm{p}=0.36$ and $\mathrm{p}=0.59)$. The combined prevalence of $P$. vivax and undetermined Plasmodium parasites ( $\mathrm{p}<0.001)$ was also correlated with $P$. vivax incidence. This was done as previous surveys found approximately 90\% of undetermined Plasmodium species detected by uPCR in western Cambodia were later identified as $P$. vivax during 12 months of prospective follow-up (Additional file 1: Figure S1) [14] (Figs. 3, 4).

\section{Village malaria worker treatment records}

Among all clinical malaria cases reported by VMWs, $154(12.7 \%)$ were among people aged 45 years and above, 842 (69.6\%) aged 15-45, 189 (15.6\%) aged five to 15 , and $(2.1 \%)$ aged $<5$ years. People recorded as migrants (no permanent address in the village) accounted for $(297 / 634) 43.9 \%$ of all malaria cases in 2013 , and (201/523) $38.4 \%$ in $2014 ; 914 / 1,210(75.5 \%)$ of subjects were male and 295/1,210 (24.5\%) were female. There was an approximately three-fold reduction in the number of $P$. falciparum and mixed $P$. falciparum and $P$. vivax cases from $88(13.9 \%)$ and 237 (37.4\%) in 2013, to $36(7.3 \%)$ and $66(13.0 \%)$ in 2014, respectively. The number and proportion of $P$. vivax cases increased from 309 (48.7\%) in 2013 to 417 (79.7\%) in 2014 respectively (Fig. 5).

\section{Discussion}

Spatial heterogeneity and risk factors for parasitaemia Variations observed in the prevalence of malaria in the study villages were consistent with a recent study in the northeast of Cambodia, which also found evidence of heterogeneity of malaria prevalence in nearby villages.

In the low season nearly one in ten people had malaria parasites. Sub-clinical vivax parasitaemia had a higher prevalence than sub-clinical falciparum parasitaemia. The predominance of $P$. vivax in the infections detected in the survey is consistent with local treatment records. 

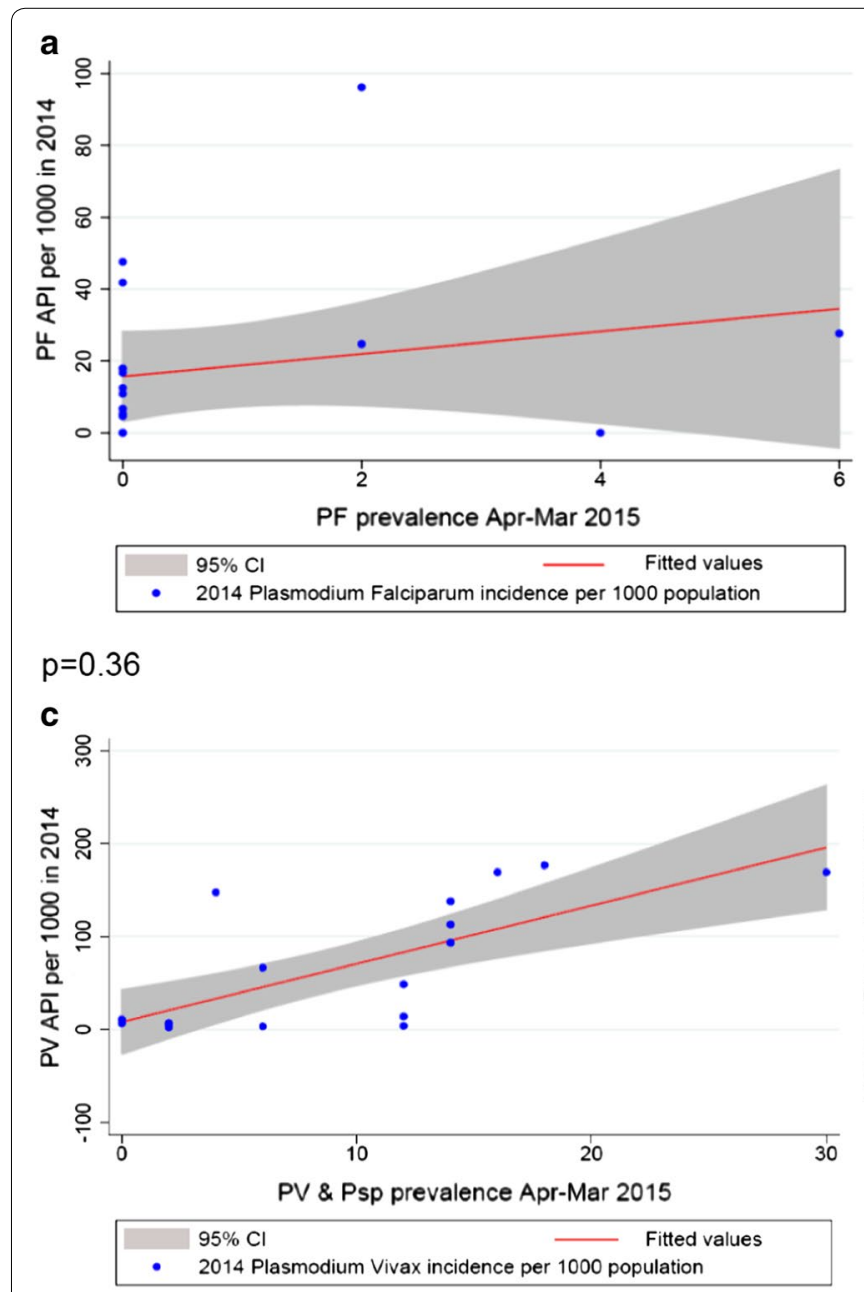

$p=0.0003$

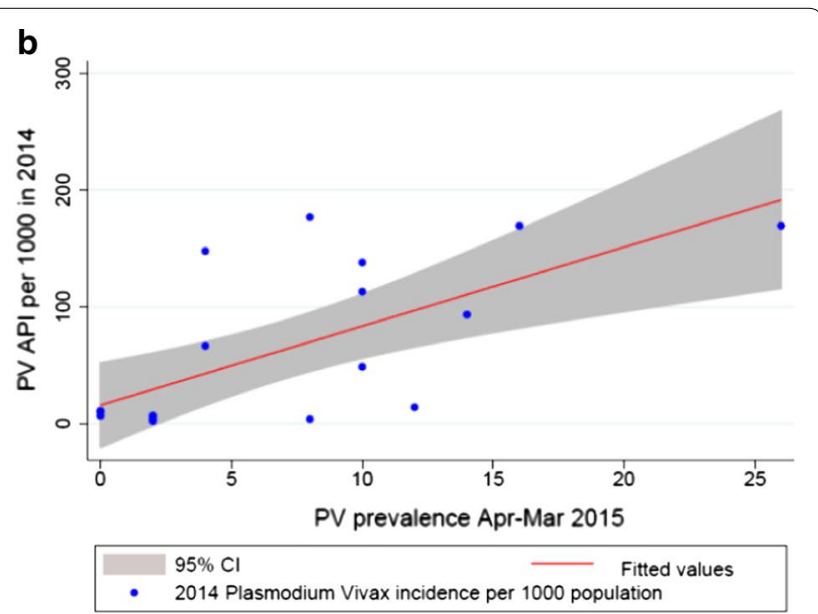

$p=0.001$

d

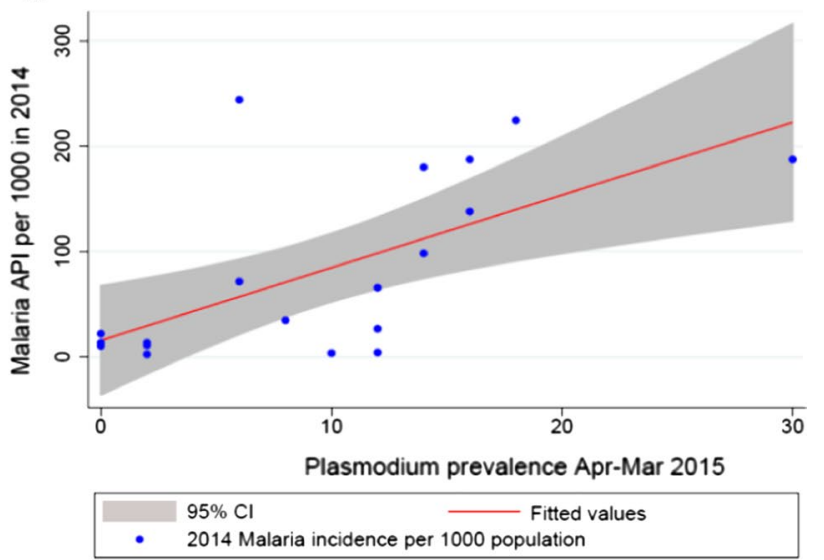

$p=0.0033$

Fig. 3 Plasmodium species prevalence by UPCR in 2015 and incidence of malaria cases in 2014. a Plasmodium falciparum prevalence by uPCR and falciparum malaria and mixed malaria incidence 2014; b Plasmodium vivax prevalence by uPCR and vivax malaria incidence 2014; c Plasmodium vivax and undetermined Plasmodium species prevalence by UPCR and vivax malaria incidence 2014; d Plasmodium prevalence by uPCR and malaria incidence 2014. In each graph, $X$ axis represents Plasmodium prevalence by uPCR during March-April 2015 and $Y$ axis represents malaria cases per 1000 population in a 1-year period. API annual parasite incidence. API = (confirmed cases during 1 year/population under surveillance) $\times 1000$. In $2014,{ }^{*} 19$ out of 20 villages malaria incidence rates were available from Battambang Provincial Health Department and are included in the analyses, except MKO

A significant proportion of clinical falciparum cases were among migrants. More detailed information on travel history and places of overnight stay in malaria-endemic areas would be helpful to better explain where malaria infections were acquired.

Young men were at greater risk of clinical malaria and had a higher prevalence of sub-microscopic malaria parasites compared to older men or to adult women. Young men were also more likely to spend time in forests and use ITNs irregularly or not at all, consistent with other studies in Cambodia [20, 21].

Plasmodium vivax prevalence determined by uPCR significantly correlated with vivax malaria incidence in each of 2014 and 2015, whereas no relationship was observed for $P$. falciparum. Due to the shorter duration of $P$. falciparum infections, it is unlikely that a single snapshot of $P$. falciparum by uPCR during the dry season would be able to describe the picture of $P$. falciparum infections throughout the whole year. Whereas for $P$. vivax, the higher persistence of sub-clinical infections means a survey in the dry season reveals a large reservoir of infections. In a previous longitudinal study a large proportion of sub-clinical $P$. vivax persisted through the year and seasonality had little effect on the number of detectable infections [14]. Even with testing a relatively small sample of each village's population, there was a 

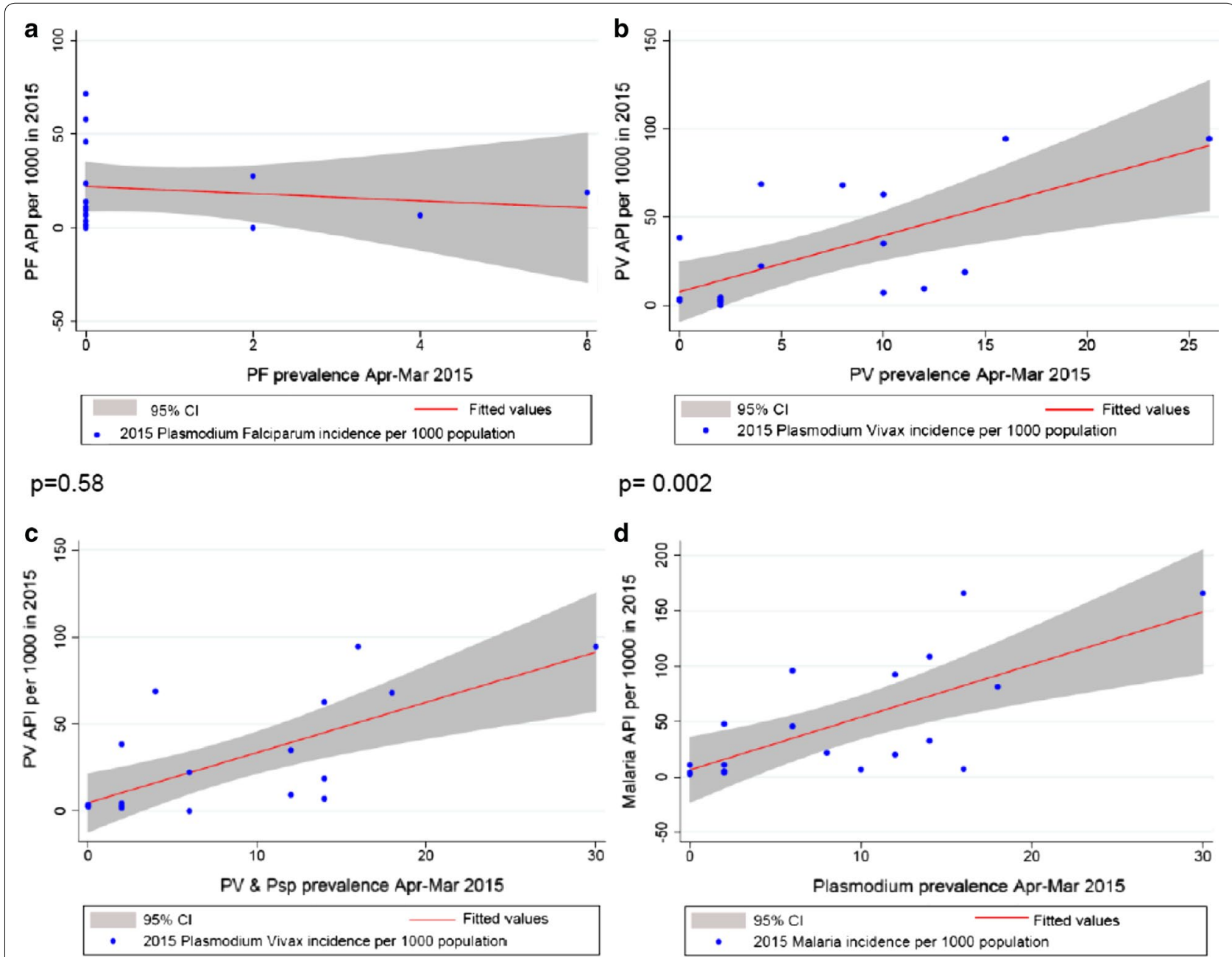

$p=0.0006$

$p=0.0008$

Fig. 4 Plasmodium species prevalence by uPCR and incidence of malaria cases in 2015. a Plasmodium falciparum prevalence by uPCR and falciparum or mixed malaria incidence 2015; b Plasmodium vivax prevalence by uPCR and vivax Malaria incidence 2015; c Plasmodium vivax and undetermined Plasmodium species prevalence by uPCR and vivax malaria incidence 2015; d Plasmodium prevalence by UPCR and malaria incidence 2015. In each graph, $X$ axis represents Plasmodium prevalence by uPCR during March-April 2015 and $Y$ axis represents malaria cases per 1000 population in a 1-year period, API annual parasite incidence. API = (confirmed cases during 1 year/population under surveillance) $\times 1000$. In $2015,{ }^{*} 19$ out of 20 villages malaria incidence rates were available from Battambang Provincial Health Department and are included in the analyses, except for PEM

significant association between numbers with clinical and sub-clinical $P$. vivax.

\section{Implications for vivax treatment}

There was a significant association between a prior history of malaria and having vivax parasitaemia. These selfreported histories (which often dated back many years) are not a promising target to guide screening or treatment as the absolute risk difference was 3\% (12 versus 9\% overall) and two-thirds of all participants reported a history of clinical malaria. However, clinical treatment records of confirmed malaria diagnoses are a more reliable predictor of persistent vivax parasitaemia [22].

\section{Implications for falciparum elimination}

To accelerate malaria elimination, malaria control programmes in Cambodia and other countries are evaluating various strategies for mass drug administration (MDA) [11, 23-25]. Although in the dry season P. falciparum prevalence in these villages was very low, six of seven uPCR-positive $P$. falciparum infections did not have recent history of travel to another province, suggesting some ongoing local transmission. Without the contribution of migrants to the clinical malaria records Samlout District may be close to interruption of $P$. falciparum transmission, but imported cases may undermine elimination. In a recent study in neighbouring Pailin Province, 


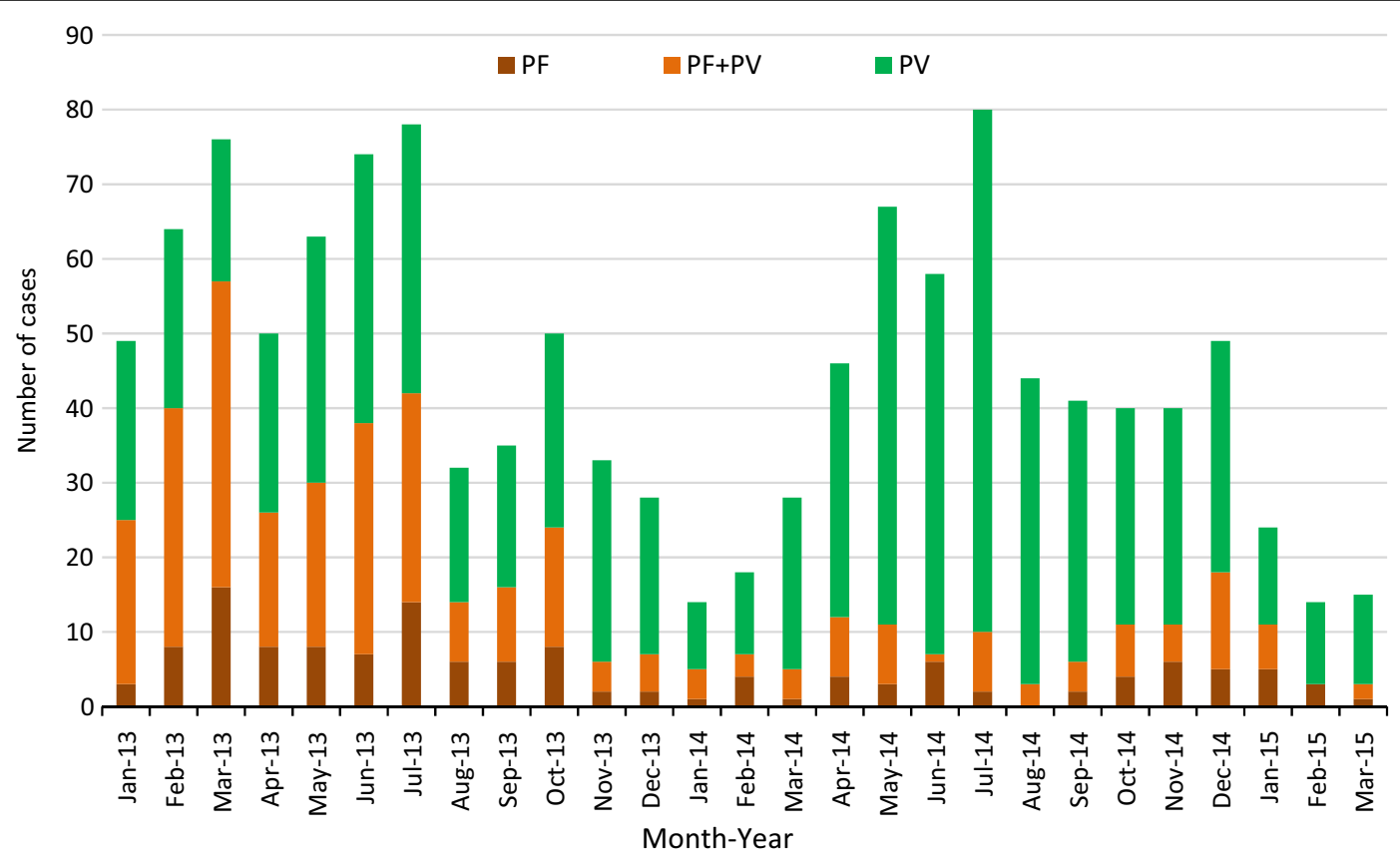

Fig. 5 Clinical malaria cases in the survey villages recorded by village malaria workers from 2013 to March 2015. Plasmodium falciparum monoinfections are red, mixed $P$. falciparum and $P$. vivax are orange, and $P$. vivax mono-infections are green

a high proportion of $P$. falciparum clinical cases were found to have C580Y Kelch mutations, and in 2013-14 prevalence surveys in Pailin, approximately half of all asymptomatic $P$. falciparum infections had markers associated with artemisinin resistance [7, 14]. In Laos, a high proportion of asymptomatic parasitaemias had the same marker associated with artemisinin resistance [26].

\section{Limitations and strengths}

The cross-sectional survey design was unable to resolve whether past clinical malaria left persistent parasites or whether these individuals were parasitaemic because they were generally at a higher risk. This study was conducted during the dry season and studies from Pailin in 2013-2014 reported a several-fold variation in $P$. falciparum prevalence over one year; therefore, it is not possible to estimate the overall contribution of these infections to transmission in these communities. It was also not possible to determine the Plasmodium species of some parasitaemias. Generally these were the participants with the lowest quantity of parasite DNA. The incidence data could be incomplete as VMW data from 2014 may have missed some people who went to other places for diagnosis and treatment.

\section{Conclusions}

The low season reservoir consisted chiefly of $P$. vivax infections with very low $P$. falciparum prevalence. Plasmodium vivax prevalence correlated with incidence of clinical malaria episodes while $P$. falciparum did not. Knowledge of the crude $P$. falciparum incidence alone was not a reliable tool to predict the hidden parasite reservoir during the dry season in this instance. More detailed knowledge of the characteristics of the $P$. falciparum cases, such as occupation and travel history could potentially distinguish indigenous and imported malaria and thereby improve the use of local treatment records to predict the sub-clinical $P$. falciparum reservoir. This can be a particularly useful tool to target malaria elimination activities such as mass screening and treatment or mass anti-malarial drug administration.

Much clinical $P$. falciparum was among migrants, forest workers or those recently returned from other provinces, suggesting transmission inside the villages themselves is now very low. Young men were more likely to have submicroscopic malaria than older men or females of any age and to exhibit behaviour that exposes them to the risk of infection (and transmission). Imported malaria has the potential to undermine provincial $P$. falciparum elimination efforts as clinical and sub-clinical infections are brought into the district by migrant workers and by local people returning from other provinces.

The spatial heterogeneity of Plasmodium prevalence between neighbouring villages shows that there is important variability in malaria epidemiology at a local level. Knowledge of the recent, detailed travel history of clinical malaria cases could provide crucial information about 
the probable source of infection and could help determine the risk of infection within individual villages. Even in villages with indigenous transmission, strategies to screen or treat sub-microscopic malaria in the dry season may be undermined by subsequent immigration of parasitaemic individuals. Therefore, targeting high-risk groups, e.g., migrant labourers, forest goers, by offering free screening and treatment, may prevent re-importation of malaria parasites from areas of higher transmission to areas in pre-elimination state and this could be an effective tool to eliminate falciparum malaria. Simultaneously, mass treatment in high transmission areas around the start of the malaria transmission season at community level may reduce the incidence of falciparum malaria significantly. It could have a collateral effect on a whole geographical area moving towards malaria elimination.

\section{Additional file}

Additional file 1: Figure S1. Malaria prevalence by UPCR and incidence per 1000 in 2015 in 16 villages.

\section{Abbreviations}

ACT: artemisinin-based combination therapy; DHA-PQP: dihydroartemisinin-piperaquine phosphate; EDTA: ethylenediaminetetraacetic acid; ITNs: insecticide-treated bed nets; MDA: mass drug administration; nested PCR: nested polymerase chain reaction; UPCR: ultrasensitive polymerase chain reaction; VMW: village malaria workers.

\section{Authors' contributions}

RT, TP, NJ, and CV conducted the study. LVS, CD and CN supervised the study. $A D$ and NJW conceived of the study and provided scientific oversight. MI, JD, $\mathrm{KP}$, and MD supervised the samples and molecular laboratory. TP, RT and RM analysed the data. RT, CV, TP, and LvS wrote the manuscript. All authors read and approved the final manuscript.

\section{Author details}

${ }^{1}$ Mahidol Oxford Tropical Medicine Research Unit, Faculty of Tropical Medicine, Mahidol University, 420/6 Rajvithi Rd, Rajthevee, Bangkok 10400, Thailand. ${ }^{2}$ Center of Tropical Medicine and Travel Medicine, Department of Infectious Diseases, Division of Internal Medicine, Academic Medical Center, University of Amsterdam, Amsterdam, The Netherlands. ${ }^{3}$ Centre for Tropical Medicine and Global Health, Nuffield Department of Clinical Medicine, University of Oxford, Oxford, UK. ${ }^{4}$ National Center for Parasitology, Entomology and Malaria Control, Phnom Penh, Cambodia. ${ }^{5}$ Provincial Health Department, Battambang, Cambodia. ${ }^{6}$ Faculty of Public Health and Policy, London School of Hygiene and Tropical Medicine, London, UK. ${ }^{7}$ World-Wide Antimalarial Resistance Network, Mahidol University, Bangkok, Thailand. ${ }^{8}$ Department of Epidemiology, Harvard T. H. Chan School of Public Health, Harvard University, Boston, USA. ${ }^{9}$ Department of Molecular Tropical Medicine and Genetics, Faculty of Tropical Medicine, Mahidol University, Bangkok, Thailand.

\section{Acknowledgements}

We thank the study participants and communities, village malaria workers and local authorities. Survey team: Chhouen Heng, Moeun Moninh, Rouen Sary, Phan Sophan, Pich Kunthea, Juum Kosal, Ly Chhean, Chan Bora, and Thida Chhuon. Laboratory team: Im Sambo, Sunninh, Chang Chan, Ngoun Savora, Buth Sophany, Cheng Samon, and Keung Chakriya. MORU Bangkok: Passathorn Sirithiranont, Jaruwan Tubprasert, Jeremy Chalk, Phaik Yeong Chea, Cholrawee Promnarate, Patrick Hannay. CNM Phnom Penh: Huy Rekol and Pengby Ngor. University of Amsterdam: Martin Grobuch. RT's PhD is funded by The Wellcome Trust, UK.

\section{Competing interests}

The authors declare that they have no competing interests.

\section{Availability of data and materials}

The dataset is available on request from $\mathrm{Dr}$ Chea Nguon, Deputy Director of the National Centre for Parasitology, Entomology and Malaria Control, Phnom Penh, Cambodia. E-mail: cheanguoncnm@gmail.com.

\section{Ethical approval}

Ethics approval was obtained from the National Ethics Committee for Health Research Cambodia (NECHR 0042), the Oxford Tropical Research Ethics Committee (OXTREC; 1017-13), and the study was registered on clinicaltrials.gov (NCT01872702)

\section{Funding}

The Wellcome Trust-Mahidol University-Oxford Tropical Medicine Research Programme is funded by the Wellcome Trust of Great Britain (reference 101148/Z/13/Z). This study was funded by the Bill and Melinda Gates Foundation BMGF OPP1081420.

Received: 27 November 2016 Accepted: 19 January 2017

Published online: 31 January 2017

\section{References}

1. Maude RJ, Nguon C, Ly P, Bunkea T, Ngor P, Canavati de la Torre SE. Spatial and temporal epidemiology of clinical malaria in Cambodia 2004-2013. Malar J. 2014;13:385.

2. Verdrager J. Epidemiology of the emergence and spread of drug-resistant falciparum malaria in South-East Asia and Australasia. J Trop Med Hyg. 1986;89:277-89.

3. Noedl H, Se Y, Schaecher K, Smith BL, Socheat D, Fukuda MM, et al. Evidence of artemisinin-resistant malaria in western Cambodia. N Engl J Med. 2008;359:2619-20.

4. Dondorp AM, Nosten F, Yi P, Das D, Phyo AP, Tarning J, et al. Artemisinin resistance in Plasmodium falciparum malaria. N Engl J Med. 2009;361:455-67.

5. Amaratunga C, Sreng S, Suon S, Phelps ES, Stepniewska K, Lim P, et al. Artemisinin-resistant Plasmodium falciparum in Pursat Province, western Cambodia: a parasite clearance rate study. Lancet Infect Dis. 2012;12:851-8.

6. Amaratunga C, Lim P, Suon S, Sreng S, Mao S, Sopha C, et al. Dihydroartemisinin-piperaquine resistance in Plasmodium falciparum malaria in Cambodia: a multisite prospective cohort study. Lancet Infect Dis. 2016;16:357-65.

7. Ashley EA, Dhorda M, Fairhurst RM, Amaratunga C, Lim P, et al. Spread of artemisinin resistance in Plasmodium falciparum malaria. N Engl J Med. 2014;371:411-23.

8. White NJ. Artemisinin resistance-the clock is ticking. Lancet. 2010;376:2051-2.

9. Dondorp AM, Fairhurst RM, Slutsker L, Macarthur JR, Breman JG, Guerin PJ, et al. The threat of artemisinin-resistant malaria. N Engl J Med. 2011;365:1073-5.

10. Maude RJ, Pontavornpinyo W, Saralamba S, Aguas R, Yeung S, Dondorp AM, et al. The last man standing is the most resistant: eliminating artemisinin-resistant malaria in Cambodia. Malar J. 2009;8:31.

11. Maude RJ, Socheat D, Nguon C, Saroth P, Dara P, Li G, et al. Optimising strategies for Plasmodium falciparum malaria elimination in Cambodia: primaquine, mass drug administration and artemisinin resistance. PLoS ONE. 2012;7:e37166.

12. WHO. Global Plan for Artemisinin Resistance Containment (GPARC). Geneva: World Health Organization; 2011. http://www.who.int/malaria/ publications/atoz/9789241500838/en/. Accessed Jan 2011.

13. Imwong M, Nguyen TN, Tripura R, Peto TJ, Lee SJ, Lwin KM, et al. The epidemiology of subclinical malaria infections in South-East Asia: findings from cross-sectional surveys in Thailand-Myanmar border areas, Cambodia, and Vietnam. Malar J. 2015;14:381.

14. Tripura R, Peto TJ, Chalk J, Lee SJ, Sirithiranont P, Nguon C, et al. Persistent Plasmodium falciparum and Plasmodium vivax infections in a western 
Cambodian population: implications for prevention, treatment and elimination strategies. Malar J. 2016;15:181.

15. Imwong M, Hanchana S, Malleret B, Rénia L, Day NP, Dondorp A, et al. High throughput ultra-sensitive molecular techniques to quantity low density malaria parasitaemias. J Clin Microbiol. 2014;52:3303-9.

16. Steenkeste N, Rogers WO, Okell L, Jeanne I, Incardona S, Duval L, et al. Sub-microscopic malaria cases and mixed malaria infection in a remote area of high malaria endemicity in Rattanakiri province, Cambodia: implication for malaria elimination. Malar J. 2010;9:108.

17. Okell LC, Ghani AC, Lyons E, Drakeley CJ. Submicroscopic infection in Plasmodium falciparum-endemic populations: a systematic review and meta-analysis. J Infect Dis. 2009;200:1509-17.

18. Shekalaghe SA, Bousema JT, Kunei KK, Lushino P, Masokoto A, Wolters $L R$, et al. Submicroscopic Plasmodium falciparum gametocyte carriage is common in an area of low and seasonal transmission in Tanzania. Trop Med Int Health. 2007;12:547-53.

19. Bhumiratana A, Intarapuk A, Sorosjinda-Nunthawarasilp P, Maneekan $P$, Koyadun S. Border malaria associated with multidrug resistance on Thailand-Myanmar and Thailand-Cambodia borders: transmission dynamic, vulnerability, and surveillance. Biomed Res Int. 2013;2013:363417.

20. Incardona S, Vong S, Chiv L, Lim P, Nhem S, Sem R, et al. Large-scale malaria survey in Cambodia: novel insights on species distribution and risk factors. Malar J. 2007;6:37.
21. Sluydts V, Heng S, Coosemans M, Van Roey K, Gryseels C, Canier L, et al. Spatial clustering and risk factors of malaria infections in Ratanakiri Province, Cambodia. Malar J. 2014;13:387.

22. Peto TJ, Kloprogge SE, Tripura R, Nguon C, Sanann N, Yok S, et al. History of malaria treatment as a predictor of subsequent subclinical parasitaemia: a cross-sectional survey and malaria case records from three villages in Pailin, western Cambodia. Malar J. 2016:15:240.

23. Song J, Socheat D, Tan B, Dara P, Deng C, Sokunthea S, et al. Rapid and effective malaria control in Cambodia through mass administration of artemisinin-piperaquine. Malar J. 2010;9:57.

24. WHO. Strategy for Malaria Elimination in the Greater Mekong Subregion 2015-30. Geneva: World Health Organization; 2015. http://www.wpro. who.int/mvp/documents/strat_mal_elim_gms/en/. Accessed 26 March 2015.

25. Seidlein L, Dondorp A. Fighting fire with fire: mass antimalarial drug administrations in an era of antimalarial resistance. Expert Rev Anti Infect Ther. 2015;13:715-30.

26. Phommasone K, Adhikari B, Henriques G, Pongvongsa T, Phongmany $P$, von Seidlein $\mathrm{L}$, et al. Asymptomatic Plasmodium infections in 18 villages of southern Savannakhet Province, Lao PDR (Laos). Malar J. 2016;15:296.

\section{Submit your next manuscript to BioMed Central and we will help you at every step:}

- We accept pre-submission inquiries

- Our selector tool helps you to find the most relevant journal

- We provide round the clock customer support

- Convenient online submission

- Thorough peer review

- Inclusion in PubMed and all major indexing services

- Maximum visibility for your research

Submit your manuscript at www.biomedcentral.com/submit 